\title{
Image reconstruction techniques applied to nuclear mass models
}

\author{
Irving O. Morales, ${ }^{1}$ P. Van Isacker, ${ }^{2}$ V. Velazquez, ${ }^{3}$ J. Barea, ${ }^{4}$ J. Mendoza-Temis,,${ }^{1}$ J. C. López Vieyra, ${ }^{1}$ \\ J. G. Hirsch, ${ }^{1}$ and A. Frank ${ }^{1}$ \\ ${ }^{1}$ Instituto de Ciencias Nucleares, Universidad Nacional Autónoma de México, 04510 México, D.F., Mexico \\ ${ }^{2}$ Grand Accélérateur National d'Ions Lourds, CEA/DSM-CNRS/IN2P3, Boîte Postale 55027, F-14076 Caen Cedex 5, France \\ ${ }^{3}$ Facultad de Ciencias, Universidad Nacional Autónoma de México, 04510 México, D.F., Mexico \\ ${ }^{4}$ Instituto de Estructura de la Materia, Consejo Superior de Investigaciones Científicas, Unidad Asociada al Departamento de Física \\ Atómica, Molecular y Nuclear, Facultad de Física, Universidad de Sevilla, Apartado 1065, E-41080 Sevilla, Spain
}

(Received 14 April 2009; revised manuscript received 11 January 2010; published 5 February 2010)

\begin{abstract}
A new procedure is presented that combines well-known nuclear models with image reconstruction techniques. A color-coded image is built by taking the differences between measured masses and the predictions given by the different theoretical models. This image is viewed as part of a larger array in the $(N, Z)$ plane, where unknown nuclear masses are hidden, covered by a "mask." We apply a suitably adapted deconvolution algorithm, used in astronomical observations, to "open the window" and see the rest of the pattern. We show that it is possible to improve significantly mass predictions in regions not too far from measured nuclear masses.
\end{abstract}

DOI: 10.1103/PhysRevC.81.024304

PACS number(s): 21.10.Dr, 42.30.Wb

\section{INTRODUCTION}

Nuclei are particularly complex systems, ranging from a single proton to more than three hundred nucleons; too large for a detailed microscopic treatment but too small for statistical methods. Interest in them is not restricted to nuclear physicists because the atomic nuclei that constitute our world are manufactured inside stars, defining their evolution and fate. Arguably, the most basic property of a nucleus is its mass. Understanding nuclear masses provides a test of our basic knowledge of nuclear structure and is an essential ingredient of the fundamental astrophysical problem of nucleosynthesis, which often takes place in far-from-stability conditions, on ultrashort time scales [1]. Though great progress has been made in measuring the mass of short-lived nuclei that are far from the region of stable, naturally occurring isotopes, theory is needed to predict their properties and guide experiments that search, for example, for regions of increased stability [2]. Despite the efforts invested in developing techniques that can accurately describe these masses, the predictions made by different models often turn out to be disconcertingly different, even in regions close to known masses. Reliable theoretical models and methodologies that can predict the mass and other properties of these "exotic" nuclei are still missing [3].

The efforts to calculate nuclear masses have been hampered by the absence of a true effective theory of the nuclear interaction and by the difficulties inherent to quantum manybody calculations. Instead, simplified approaches to model the atomic nucleus have been devised. The cornerstone of nuclear mass models is the semi-empirical mass formula proposed by von Weizsäcker [4] and Bethe and Bacher [5] in the 1930s, which is based on a picture of the nucleus as a uniform, very dense, charged liquid drop-the so-called liquid-drop model (LDM) — which gives an accurate overall description of nuclear masses, but lacks some quantum-mechanical effects due to shell closures, pairing, quadrupole correlations, etc. Thus, large deviations from the measured masses are observed in certain regions of the nuclear chart. The inclusion of these effects usually requires other treatments, like the finite-range droplet model [6], which combines macroscopic effects with microscopic shell and pairing corrections, the model of Duflo and Zuker [7], and the Hartree-Fock approach [8]. In general, however, it is difficult to match theory and experiment (for all known nuclei) with an average precision better than about $0.5 \mathrm{MeV}$ [3]. More troublesome is the fact that different model predictions tend to diverge from each other for regions of nuclei with unknown mass.

\section{SYSTEMATIC PATTERNS}

The starting point in the proposed approach is the striking color patterns in the $(N, Z)$ plane shown in Fig. 1, which result after taking the difference between the experimentally known masses [9] and predictions of several models: an improved macroscopic LDM [10], an upgrade of the LDM by the inclusion of schematic microscopic shell corrections [10] (LDMM), the 31-parameter Duflo-Zuker (DZ) model [7], and the GarveyKelson (GK) relations [11]. For each model, we observe a residual systematic pattern related to the physics and that is not included in the model. Thus, for example, the LDM does not contain microscopic information on independent-particle motion, and consequently the pattern shows systematic deviations related to shell closures, nuclear deformations, and residual interactions in a compelling graphic form [see Fig. 1(a)]. The microscopic behavior can be parametrized by the inclusion of a schematic microscopic shell correction [10]:

$$
\begin{aligned}
B E_{\mathrm{LDMM}}(N, Z) \equiv & B E_{\mathrm{LDM}}(N, Z) \\
& -a_{\mathrm{f}} F_{\max }+a_{\mathrm{ff}} F F_{\max }+a_{\mathrm{const}},
\end{aligned}
$$

where $B E_{\mathrm{LDM}}$ is the nuclear binding energy as obtained in the LDM and

$$
\begin{aligned}
F_{\max } & =\frac{n_{\nu}+n_{\pi}}{2}-\left\langle\frac{n_{\nu}+n_{\pi}}{2}\right\rangle, \\
F F_{\max } & =\left(\frac{n_{v}+n_{\pi}}{2}\right)^{2}-\left\langle\left(\frac{n_{v}+n_{\pi}}{2}\right)^{2}\right\rangle,
\end{aligned}
$$



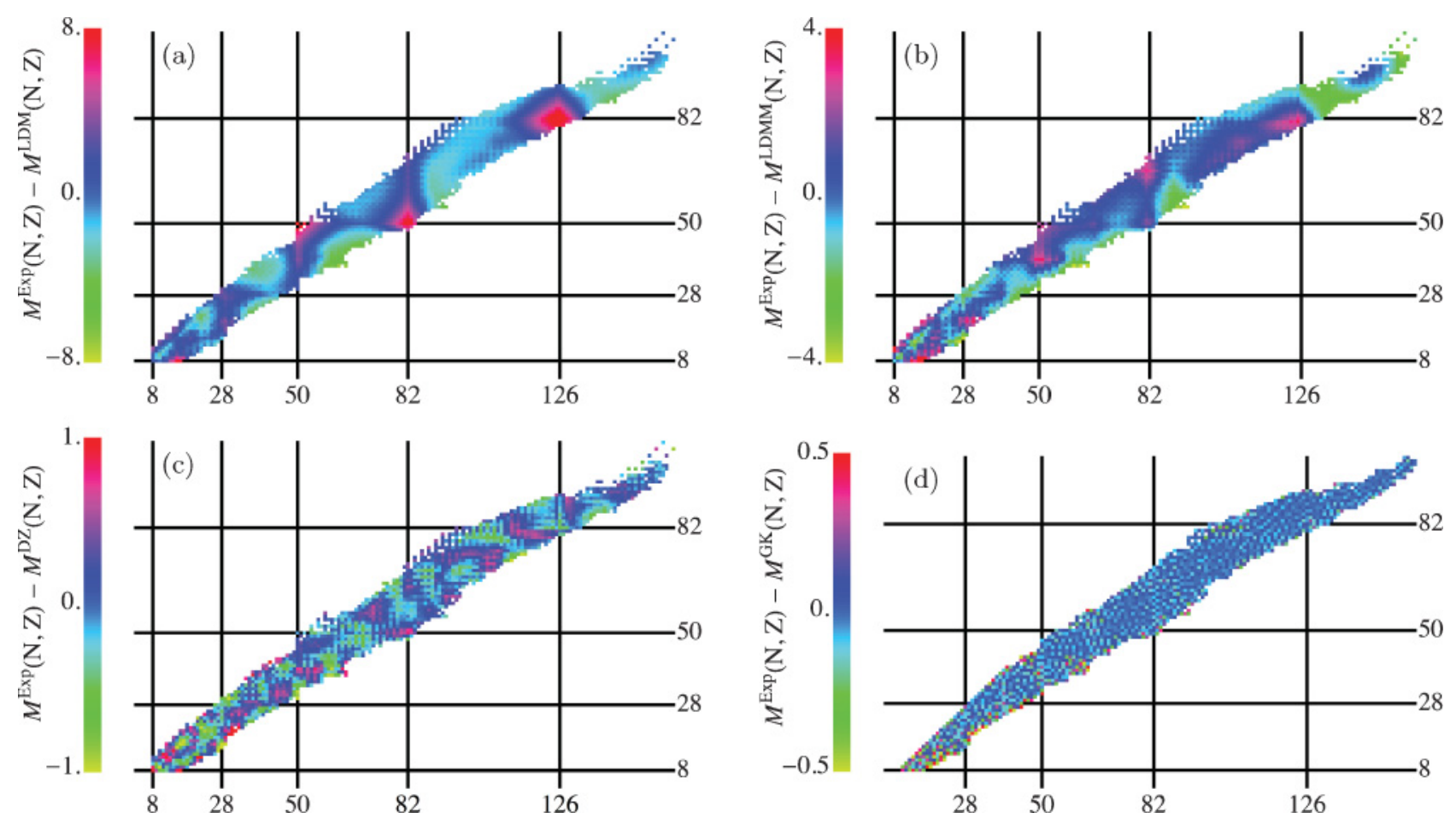

FIG. 1. (Color online) Differences between experimental masses and those calculated with the four models: (a) the liquid-drop model (LDM), (b) the liquid-drop model with schematic shell correction (LDMM), (c) the Duflo-Zuker model (DZ), and (d) the Garvey-Kelson relations (GK).

are linear and quadratic terms in the numbers of valence neutron $n_{v}$ (proton $n_{\pi}$ ) particles or holes, counted from the nearest closed shell, and the quantities in brackets are their average values. The constant $a_{\text {const }}$, used to re-center the prediction average (see Ref. [10]), and $a_{\mathrm{f}}$ and $a_{\mathrm{ff}}$ are parameters found by fitting the experimental masses. Although this parametrization still leads to large errors, it is able to schematically reproduce the main trends observed in the mass surface near the shell closures. These terms are similar to the ones used in parametrizations proposed by R. F. Casten [12]. The inclusion of these terms reduces the rms deviation but some systematic patterns still appear, as is shown in Fig. 1(b) (note the change of scale). Finally, if we take a more complex approach such as the DZ model, we still observe small but systematically correlated errors [Fig. 1(c)]. The remaining patterns suggest physics that have not been incorporated into the models. Figure 1(d) displays the difference between experimental masses and masses obtained from the GK relations [11]. The remaining deviations are, in this case, close to white noise, which shows that it is in principle possible to predict masses to a significantly better precision and, therefore, that it is worthwhile to attempt to take into account the missing physical effects (see, however, Ref. [13]).

\section{IMAGE RECONSTRUCTION}

The patterns shown in Fig. 1 suggest an approach to mass prediction based on image reconstruction techniques. The basic idea is to consider that the more than 2000 differences between the different models and the known nuclear masses represent partial views of a larger image array in the $(N, Z)$ plane, and that all other mass differences (perhaps around 7000 in number) that exist between the neutron and proton drip lines remain hidden, covered by a "mask." Thus, the question is whether we can "open the window" to unveil the remaining pattern, or at least part of it. We show here that indeed this information can be reliably uncovered, at least for regions not too far from measured masses, with image reconstruction techniques. The success of the approach strongly depends upon whether the physical features associated with the changing patterns in the nuclear landscape can be coded in terms of (global) regularities in the $(N, Z)$ plane, and whether they can in turn be modeled by a finite number of harmonic components. An important assumption in this approach is that the observed regularity and relative smoothness of the mass-difference landscape remains throughout [14]. Although substantial changes in mass differences may occur in a rather short interval (e.g., because of shell structure or shape transitions), there are no sudden jumps from one nucleus to another. Figure 1(a) shows that, to a good approximation, the landscape can be considered to vary smoothly as a function of nucleon number. More precise checks of this assumption can be made, for example by testing whether the GK relations (which are a measure of smoothness) become increasingly inaccurate for predictions further away from stable nuclei. Our tests indicate that this is not the case-they remain equally accurate, even when approaching areas close to the drip lines [15]. We are thus motivated to follow this approach further. 
We display the mass-difference table by a two-dimensional array of pixels, with $N$ in the horizontal position and $Z$ in the vertical one. Differences between experimental masses $m_{\text {expt }}(N, Z)$ and calculated masses $m_{\mathrm{th}}(N, Z)$ define the color image function

$$
i(N, Z) \equiv m_{\text {th }}(N, Z)-m_{\text {expt }}(N, Z) .
$$

The relationship between $i(N, Z)$ and the full pattern $m(N, Z)$ is then given by

$$
i(N, Z)=m(N, Z) \cdot w(N, Z),
$$

where $w(N, Z)$ is a binary mask function, taking the value 1 for positions $(N, Z)$ where nuclear masses are known, and 0 otherwise (although a "weighted" mask may be used insteadsee Ref. [16]). We thus need to extract $m(N, Z)$ from Eq. (3). If $I\left(k_{N}, k_{Z}\right), M\left(k_{N}, k_{Z}\right)$, and $W\left(k_{N}, k_{Z}\right)$ are the Fourier transforms of $i(N, Z), m(N, Z)$, and $w(N, Z)$, respectively, then

$$
I\left(k_{N}, k_{Z}\right)=M\left(k_{N}, k_{Z}\right) * W\left(k_{N}, K_{Z}\right),
$$

where $M * W$ is the convolution of the functions $M$ and $W$. Since both $i(N, Z)$ and $w(N, Z)$ are known for the entire domain, their Fourier transforms $I\left(k_{N}, k_{Z}\right)$ and $W\left(k_{N}, k_{Z}\right)$ can be evaluated directly. The problem is narrowed down to obtaining the function $M\left(k_{N}, k_{Z}\right)$, from which $m(N, Z)$ can be recovered by applying an inverse Fourier transform. Formally, this is a deconvolution problem. Deconvolution is nontrivial and may lead to non-unique solutions, but there exist several algorithms, such as the CLEAN method, often used in radio astronomy [17], and the maximum entropy method [18], which provide testable methodologies. We have chosen a specially adapted version of the CLEAN algorithm used in the reconstruction of texture patches [19].

The main assumption of the CLEAN method is that the remaining patterns shown in Fig. 1 can be modeled by a finite number of harmonic components. The Fourier spectrum of the known data $I\left(k_{N}, k_{Z}\right)$ is viewed as a corrupt version of the Fourier spectrum of the complete data $M\left(k_{N}, k_{Z}\right)$. This corruption is due to the mask and the main goal is to remove this noise and construct a clean Fourier spectrum, choosing those components that best explain the observed patterns in the image.

The uncorrupted version of the spectrum is made of components such as

$$
T\left(k_{N}, k_{Z}\right)=a \delta\left(k_{N}-u, k_{Z}-v\right)+a^{*} \delta\left(k_{N}+u, k_{Z}+v\right),
$$

where $a$ is the complex amplitude of the component and $(u, v)$ is the position in Fourier space. The effect of the mask on this component is

$$
I\left(k_{N}, k_{Z}\right)=T\left(k_{N}, k_{Z}\right) * W\left(k_{N}, K_{Z}\right),
$$

and by expanding the convolution we obtain

$$
I\left(k_{N}, k_{Z}\right)=a W\left(k_{N}-u, k_{Z}-v\right)+a^{*} W\left(k_{N}+u, k_{Z}+v\right),
$$

so that this single component becomes distorted. The form of the mask guarantees that the dominant peak of its Fourier spectrum is the $\mathrm{DC}$ or constant term $(u, v=0)$ and, because of this, the position remains unaltered, only the amplitude is changed due to the mask, and the corruption of the mask appears as additional smaller peaks around the component. To achieve the deconvolution it is necessary to determine the nature of the original component, namely the true amplitude and position $(a, u, v)$ given the corrupted Fourier spectrum $I\left(k_{N}, k_{Z}\right)$ and the Fourier spectrum of the mask $M\left(k_{N}, k_{Z}\right)$.

Because the position of the true components remains the same and the spurious peaks are smaller than the principal ones, we can find the principal component of the data by locating the major pair in $I\left(k_{N}, k_{Z}\right)$. Substitution of $k_{N}=u$ and $k_{Z}=v$ in Eq. (7) gives

$$
I(u, v)=a W(0,0)+a^{*} W(2 u, 2 v),
$$

which we can rearrange using the conjugate into

$$
a=\frac{I(u, v) W(0,0)-I^{*}(u, v) W(2 u, 2 v)}{W(0,0)^{2}-W(2 u, 2 v) W^{*}(2 u, 2 v)} .
$$

Thus, we can recover the true amplitude of the original harmonic component $T\left(k_{N}, k_{Z}\right)$ and the true nature of the component is thus obtained for both the position and amplitude.

The CLEAN algorithm essentially proceeds by sequentially finding the frequency position of the peak with maximal intensity in the corrupted Fourier spectrum. Once this peak is located, the true amplitude is calculated with Eq. (9) and a clean version of the Fourier spectrum is updated. This clean spectrum is constructed placing the component in the true frequency and with the correct amplitude before proceeding to the next iteration. A new version of the corrupted spectrum is calculated eliminating the removed component and the effects produced on it by the mask. The new corrupted spectrum is called the residual spectrum.

If $R^{i}\left(k_{N}, k_{Z}\right)$ is the $i$ th residual and $R^{0}\left(k_{N}, k_{Z}\right)=$ $I\left(k_{N}, k_{Z}\right)$, the CLEAN algorithm consists of the following steps:

(i) Locate the position $\left(u^{i}, v^{i}\right)$ of the $i$ th clean component from the maximum of $R^{i-1}\left(k_{N}, k_{Z}\right)$.

(ii) Calculate the true amplitude with Eq. (9).

(iii) Generate the $i$ th residual spectrum

$$
\begin{aligned}
R^{i}\left(k_{N}, k_{Z}\right)= & R^{i-1}\left(k_{N}, k_{Z}\right) \\
& -\left(a^{i} W\left(k_{N}-u^{i}, k_{Z}-v^{i}\right)\right. \\
& \left.+a^{*} W\left(k_{N}+u^{i}, k_{Z}+v^{i}\right)\right) .
\end{aligned}
$$

This procedure is repeated until a stopping criterion is reached at iteration $K$, after which the clean spectrum $C\left(k_{N}, k_{Z}\right)$ is constructed:

$$
\begin{aligned}
C\left(k_{N}, k_{Z}\right)= & \sum_{i=1}^{K}\left(a^{i} \delta\left(k_{N}-u^{i}, k_{Z}-v^{i}\right)\right. \\
& \left.+a^{i *} \delta\left(k_{N}+u^{i}, k_{Z}+v^{i}\right)\right) .
\end{aligned}
$$

Once the clean spectrum is constructed, the concealed image is obtained by applying the inverse Fourier transform to it. 


\section{MASS PREDICTIONS}

With the algorithm described previously it is possible to extrapolate the remaining patterns shown by the mass differences $\Delta m=m_{\text {expt }}-m_{\text {th }}$. Once this extrapolated pattern is obtained, it is then possible to predict the nuclear masses by adding the mass predicted by the model:

$$
m(N, Z)=\Delta m_{\text {extrapolated }}(N, Z)+m_{\text {th }}(N, Z) .
$$

It is in this sense that the CLEAN algorithm is able to improve on the model used to calculate the mass differences.

In order to provide a measure of the degree of improvement of the model used for the mass differences, two tests have been applied. In the first we take the set of measured masses with $N \geqslant 28, Z \geqslant 28$ from the Atomic Mass Evaluation 2003 (AME03) compilation of Audi et al. [9] and divide it into two subsets. We use the 1454 masses present in the previous AME95 compilation [20] as input to generate the pattern (by fitting the mass model's parameters using this set), and then we measure the predictability of the model by calculating mass differences for the other 371 experimental masses [21]. These predictions can subsequently be compared with the results obtained by applying CLEAN to the same input subset, as we explain below. We refer to this as the "AME95-03 test." This test has been extensively used before to measure the predictive power of nuclear mass models [3]. The second test consists of predicting the mass differences of 301 nuclei at the border of the AME03-measured landscape [21], following the same procedure as in the first test. We have applied both tests to three models with different degrees of accuracy. The first is a macroscopic LDM with 7 parameters (Eq. (2) in Ref. [10]) that are determined by fitting (a) the (updated) AME95 data for the AME95-03 test, or (b) the AME03 data without the border for the AME03-border test. The second model is an upgraded version of this macroscopic LDM, denoted by LDMM, and described in Sec. II. Finally, the third mass model used for the reconstruction is the 31-parameter DZ model [7].

In these tests the CLEAN method is iterated until a rms deviation of $100 \mathrm{keV}$ is achieved for the input data fit. Table I shows a comparison of the rms deviations for the AME95-03 test, and those obtained with the CLEAN method for each model. Table II gives a similar comparison for the AME03border test.

In the AME95-03 test the largest CLEAN improvement ( $\sim 62 \%$ rms reduction) is obtained for the LDMM of Eq. (1). The improved rms, surprisingly, is comparable with the corresponding rms in the DZ model (see Table I). For the simple LDM (Eq. (2) in Ref. [10]) we also find a large rms reduction of $\sim 54 \%$, whereas for the DZ model we find it

TABLE I. AME95-03 test: Predictability for the set of nuclei in AME03 but not in AME95, restricted to $N, Z \geqslant 28$.

\begin{tabular}{lcc}
\hline \hline Model & RMS & RMS (with CLEAN) \\
\hline LDM, Eq. (2) in [10] & $1.9307 \mathrm{MeV}$ & $0.8763 \mathrm{MeV}$ \\
LDMM, Eq. (1) & $0.9955 \mathrm{MeV}$ & $0.3718 \mathrm{MeV}$ \\
DZ model [7] & $0.3348 \mathrm{MeV}$ & $0.2727 \mathrm{MeV}$ \\
\hline \hline
\end{tabular}

TABLE II. AME03-border test: Predictability for the set of nuclei in the border of AME03, restricted to $N, Z \geqslant 28$.

\begin{tabular}{lcc}
\hline \hline Model & RMS & RMS (with CLEAN) \\
\hline LDM, Eq. (2) in [10] & $2.7763 \mathrm{MeV}$ & $0.9168 \mathrm{MeV}$ \\
LDMM, Eq. (1) & $1.9804 \mathrm{MeV}$ & $0.9333 \mathrm{MeV}$ \\
DZ model [7] & $0.4039 \mathrm{MeV}$ & $0.3133 \mathrm{MeV}$ \\
\hline \hline
\end{tabular}

to be $\sim 20 \%$. The latter, smaller improvement is expected, because this model is already in very good agreement with the experimental masses. Nevertheless, this rms reduction represents a significant correction.

A similar situation occurs for the AME03-border test. The largest improvement ( $\sim 66 \% \mathrm{rms}$ reduction) occurs for the macroscopic LDM, followed by LDMM of Eq. (1) with a $\sim 52 \%$ rms reduction. Again, as in the AME95-03 test, the rms in the DZ model decreases by $\sim 20 \%$. These results suggest that the CLEAN method can efficiently incorporate the residual patterns observed in the experimental masses. The rms, however, being an average measure, is not a sufficiently clear gauge. Therefore, we now turn to an amplified view of our results, using two-neutron separation energies $S_{2 \mathrm{n}}$.

Two-neutron separation energies, $S_{2 \mathrm{n}}(N, Z) \equiv B E(N$, $Z)-B E(N-2, Z)$, contain detailed information about nuclear structure effects. In Figs. 2, 3, and 4 we plot $S_{2 \text { n }}$ for isotope series in the $N \sim 78-128$ region for the AME95-03 test using the LDM, the LDMM of Eq. (1), and the DZ model, respectively.

The predictions of the macroscopic LDM, as shown in Fig. 2, are completely flat, with no structure at all. In contrast, the data display strong variations at the magic numbers $N=82$ and 126, and substructures near $N \sim 90$. After reconstruction, those structures are well described (with an rms of $\sim 0.1 \mathrm{MeV}$ ) in the fitted region (AME95 data). On the other hand, the benchmark AME03 data are also surprisingly well predicted (with an rms of $0.8763 \mathrm{MeV}$-see Table I). Although the shell structures are well described by the CLEAN algorithm, the reconstruction is not sufficiently constrained. This is illustrated by the presence of spurious substructures that, although small in magnitude, are not seen in the $S_{2 n}$ data. To minimize such spurious effects, it is necessary to impose additional constraints, and this can be achieved, for example, by schematically including shell correction terms as is done in the LDMM of Eq. (1).

Figure 3 shows $S_{2 \mathrm{n}}$ in the $N \sim 78-128$ region of the AME95-03 test, obtained with the LDMM [isotopic lines in Fig. 3(a)], and the results obtained after applying the reconstruction algorithm [isotopic lines in Fig. 3(b)]. In the LDMM, the magic numbers $N=82$ and 126 are incorporated into the model from the beginning. The experimental $S_{2 \mathrm{n}}$ in those regions displays discontinuities that are well described by the LDMM. However, the substructure observed in the data at $N \sim 90$ is not properly accounted for [see Fig. 3(a)]. After applying the reconstruction to LDMM, the substructure is correctly described (with an rms of $\sim 0.1 \mathrm{MeV}$ ) in the fitted region. It is remarkable that the region of the benchmark AME03 data (green bullets in Fig. 3) is also accurately 

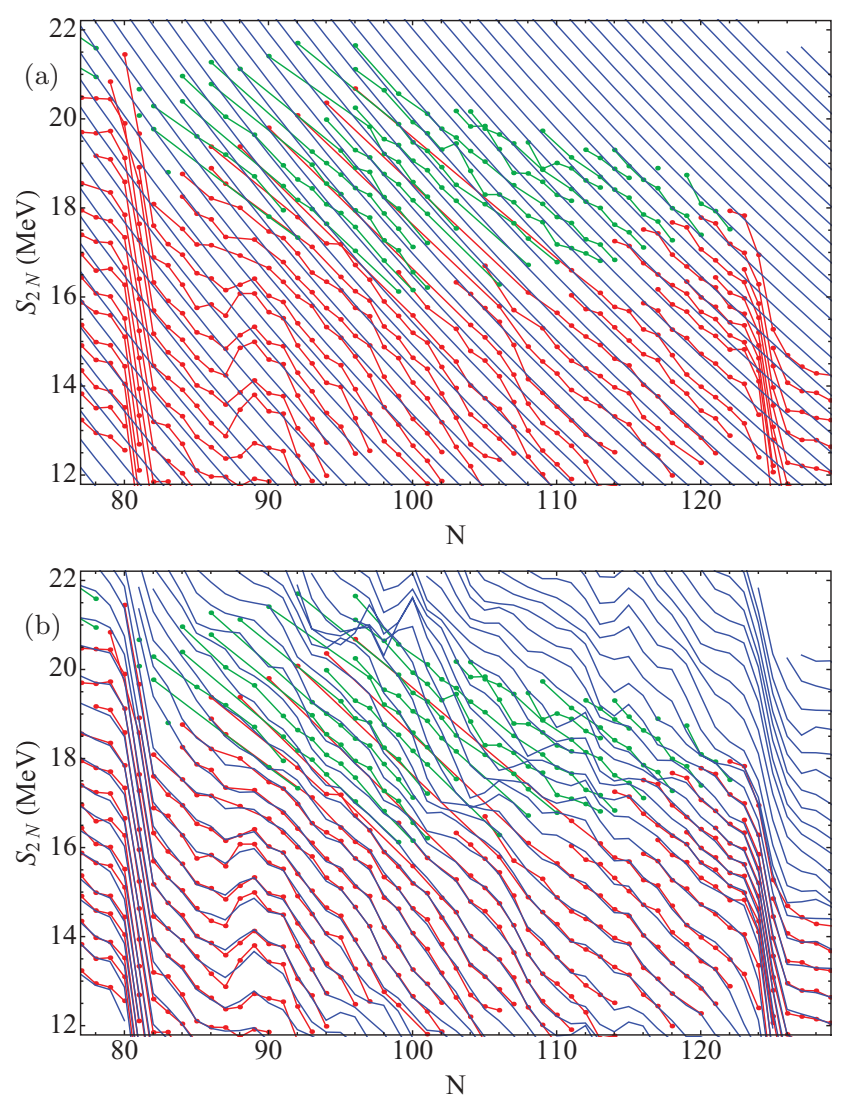

FIG. 2. (Color online) AME95-03 test: Two-neutron separation energies $S_{2 \mathrm{n}}$ isotopic lines (in blue) predicted by (a) the LDM and (b) its improvement using the CLEAN reconstruction. Red bullets indicate the input (fitted) data and green bullets indicate the benchmark (predicted) data.

predicted (with an rms of $0.3718 \mathrm{MeV}$-see Table I). The constraints imposed by the schematic shell-correction terms in the LDMM of Eq. (1) are thus sufficient to remove the spurious substructure observed in the LDM reconstruction.

The results for $S_{2 \mathrm{n}}$ obtained with the DZ model in the $N \sim$ 78-128 region of the AME95-03 test, and its improvement by the image reconstruction method, are shown in Figs. 4(a) and 4(b), respectively. In this case, the DZ model shows the presence of the substructure at $N \sim 90$, but the experimental $S_{2 \mathrm{n}}$ are not accurately described. Furthermore, the isotopic lines become flat outside the region where measurements are available. The DZ model has an rms of $0.3384 \mathrm{MeV}$ (see Table I), giving an excellent overall description of $S_{2 \mathrm{n}}$ in the predicted region. Nevertheless, the reconstruction method can be used to improve the description by a significant $\sim 20 \%$.

Figures 2, 3, and 4 show that the main effect of applying the CLEAN algorithm to the predictions of the various models is to add "texture" to the mass surface, which corresponds to some of the physical effects not included in the model.

We have made similar tests in the $N \sim 110-160$ region for the AME03-border case, using the $S_{2 \mathrm{n}}$ obtained with the LDM, LDMM, and DZ models, and the corresponding calculations using the image reconstruction method. The improvements as a result of the CLEAN procedure are of the same quality as for the AME95-03 test.
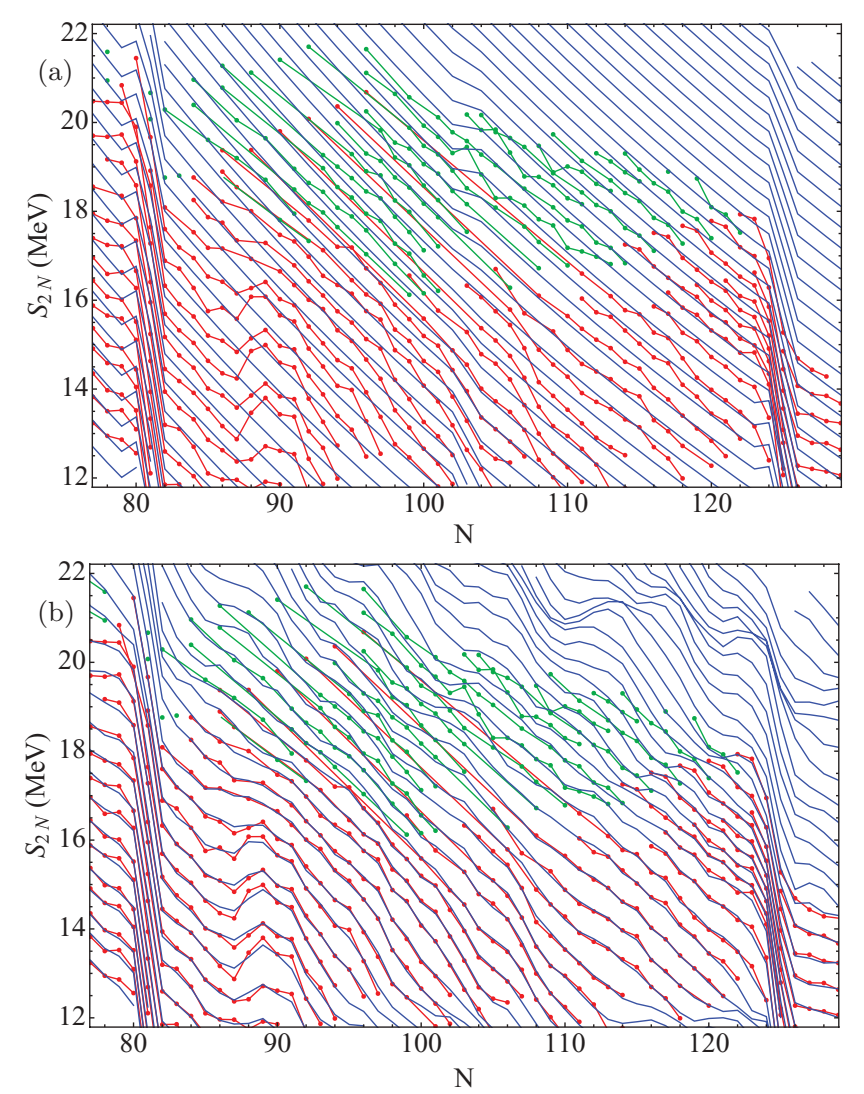

FIG. 3. (Color online) AME95-03 test: Two-neutron separation energies $S_{2 n}$ isotopic lines (in blue) predicted by (a) the LDMM and (b) its improvement using the CLEAN reconstruction. Red bullets indicate the input (fitted) data and green bullets indicate the benchmark (predicted) data.

The mass predictions obtained applying the CLEAN algorithm to the DZ model are comparable to the best available global mass predictions. However, it is of interest to also compare them with the masses recommended by Audi et al. [20], which are predicted using the systematic trends of the mass surface and its derivatives. This method provides the best short-range mass extrapolations [3], which have been published for sets of three or four nuclides in the neighborhood of those with measured masses in the Atomic Mass Evaluations [9,20]. These predictions are performed nucleus by nucleus, combining a (rather elaborate) graphical analysis with relevant physical information [9]. This procedure leads to an rms of $0.1615 \mathrm{MeV}$ for the predictions corresponding to the AME95-03 test. Since these extrapolations are more accurate than the DZ+CLEAN predictions, we expect that the masses obtained applying the CLEAN algorithm to the DZ model should be closer to the predictions of Audi et al. than the DZ masses. In order to test this we have calculated the rms deviation between the masses extrapolated by Audi $e t a l$. and the predictions of the DZ model with and without CLEAN. For the DZ model we obtain an rms of $0.3305 \mathrm{MeV}$ while for the DZ+CLEAN predictions we get an rms of $0.2694 \mathrm{MeV}$, which is significantly smaller. This shows that the DZ predictions are indeed improved using the CLEAN algorithm. 

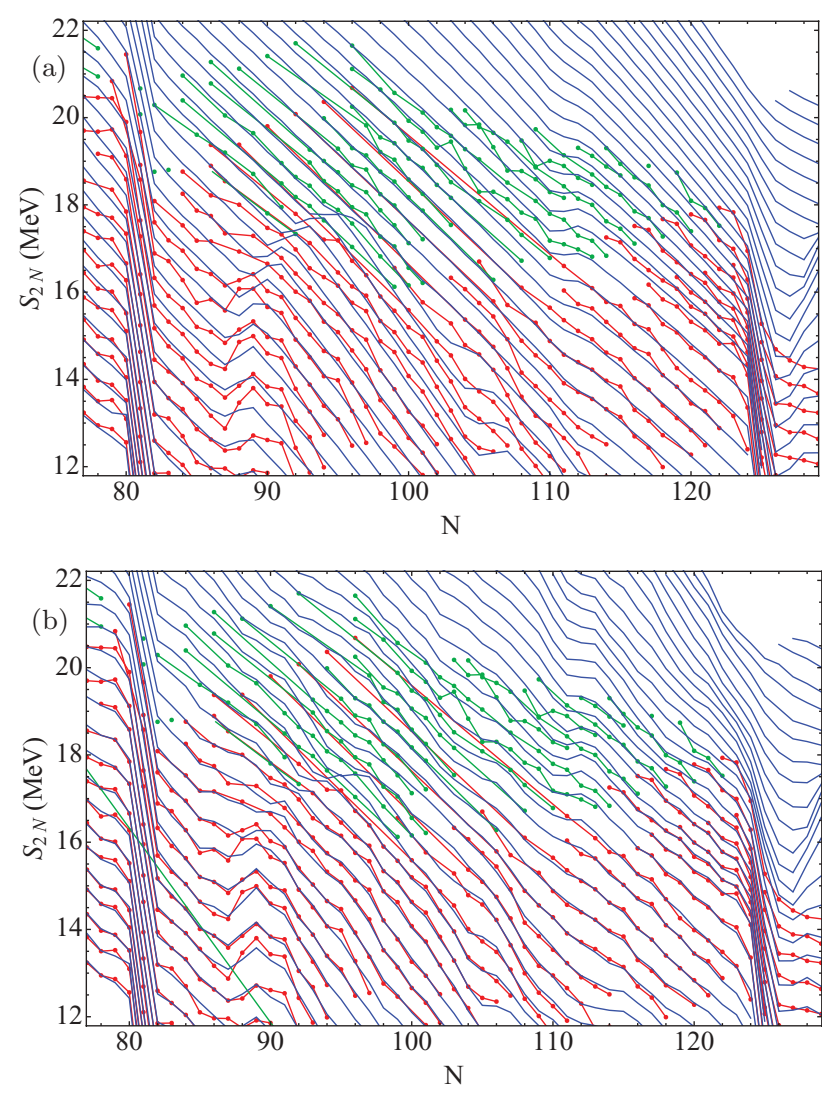

FIG. 4. (Color online) AME95-03 test: Two-neutron separation energies $S_{2 \mathrm{n}}$ isotopic lines (in blue) predicted by (a) the DZ and (b) its improvement using the CLEAN reconstruction. Red bullets indicate the input (fitted) data and green bullets indicate the benchmark (predicted) data.

\section{THE ACCURACY OF CLEAN}

The rms deviations obtained in the previous tests show that the CLEAN method improves the mass predictions of the models used as input. However, these results reflect the global behavior of CLEAN. It would be desirable to measure the accuracy of the CLEAN extrapolation as a function of the distance to the known region. However, defining such a distance is not simple.

A reliable method to obtain accurate predictions of nuclear masses in the neighborhood of a known region is based on the GK relations [11] used in an iterative procedure [13]. These relations are equations involving six neighboring masses; from the knowledge of five masses it is possible to obtain a prediction for the sixth (although there are six ways in which this can be done for each mass). The experimentally known masses accurately satisfy the GK relations and, as mentioned before, there is no evidence that this accuracy decreases away from stability. Unfortunately, because of the form of these relations it is only possible to make predictions near known regions of the nuclear chart. However, it is possible to use predicted masses as known masses and repeat the process in an iterative fashion [13]. At each iteration new masses a bit further away from the previous masses are predicted. We can use this iteration procedure as a means of defining a

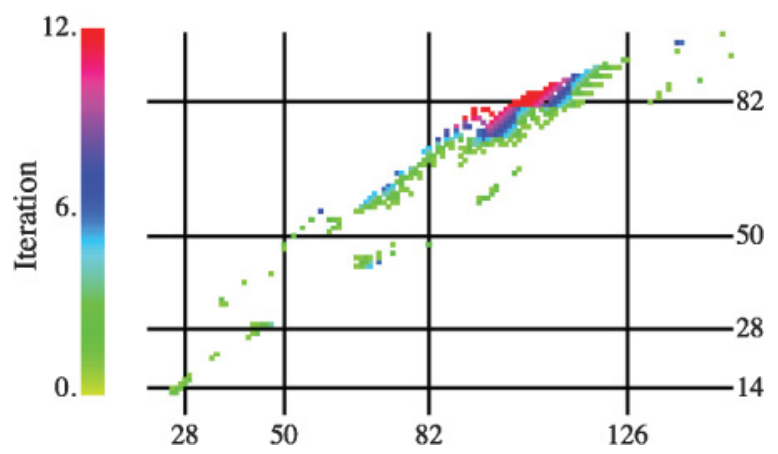

FIG. 5. (Color online) The iteration in the GK process at which each nucleus is predicted in the AME95-03 test.

distance. For example, the nuclei predicted in the first iteration are defined to be at distance one, the nuclei predicted in the second iteration are two units away, and so on. Figure 5 shows the iteration of the GK process at which each nucleus is predicted in the AME95-03 test, showing this to be a consistent measure of distance from the known region. For the border test this definition of distance is not useful because all nuclei in the prediction are as close as possible to the known region.

Two measurements of deviation can be defined that are a function of distance: $\sigma^{i}$, which is the rms for nuclei at distance $i$, and $A \sigma^{i}$, which is the rms for nuclei with distance values up to $i$ (i.e., an accumulative rms up to distance $i$ ). Figure 6 and
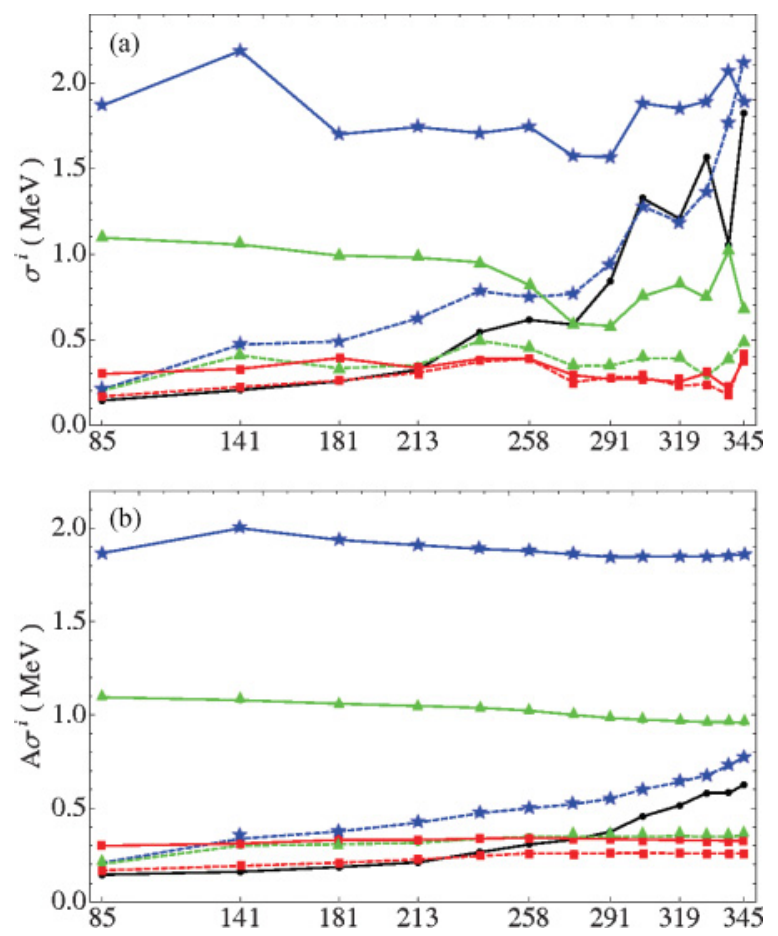

FIG. 6. (Color online) (a) $\sigma^{i}$ and (b) $A \sigma^{i}$ as a function of the number of nuclei for each unit of distance predicted in the AME95-03 test for the GK iterative procedure (black circles), LDM (blue stars), LDM improved by CLEAN (blue stars dashed), LDMM (green triangles), LDMM improved by CLEAN (green triangles dashed), DZ (red squares), and DZ improved by CLEAN (red squares dashed). 
TABLE III. $\sigma^{i}$ and $A \sigma^{i}$ deviations in MeV for the GK iterative procedure, LDM, LDM improved by CLEAN, LDMM, LDMM improved by CLEAN, DZ, and DZ improved by CLEAN for the AME95-03 test. The second column shows the number of nuclei used for calculating the rms deviations. A graphical comparison of these data is shown in Fig. 6.

\begin{tabular}{|c|c|c|c|c|c|c|c|c|}
\hline Distance & Nuclei & GK & LDM & $\begin{array}{l}\text { LDM+ } \\
\text { CLEAN }\end{array}$ & LDMM & $\begin{array}{l}\text { LDMM+ } \\
\text { CLEAN }\end{array}$ & DZ & $\begin{array}{c}\text { DZ+ } \\
\text { CLEAN }\end{array}$ \\
\hline$\sigma^{1}$ & 85 & 0.144 & 1.866 & 0.208 & 1.095 & 0.202 & 0.299 & 0.168 \\
\hline$A \sigma^{1}$ & 85 & 0.144 & 1.866 & 0.208 & 1.095 & 0.202 & 0.299 & 0.168 \\
\hline$\sigma^{2}$ & 56 & 0.205 & 2.186 & 0.471 & 1.055 & 0.407 & 0.329 & 0.224 \\
\hline$A \sigma^{2}$ & 141 & 0.161 & 1.999 & 0.338 & 1.079 & 0.301 & 0.311 & 0.192 \\
\hline$\sigma^{3}$ & 40 & 0.256 & 1.698 & 0.489 & 0.990 & 0.332 & 0.392 & 0.260 \\
\hline$A \sigma^{3}$ & 181 & 0.186 & 1.937 & 0.376 & 1.060 & 0.308 & 0.331 & 0.209 \\
\hline$\sigma^{4}$ & 32 & 0.323 & 1.741 & 0.624 & 0.978 & 0.352 & 0.334 & 0.307 \\
\hline$A \sigma^{4}$ & 213 & 0.211 & 1.909 & 0.423 & 1.048 & 0.315 & 0.331 & 0.226 \\
\hline$\sigma^{5}$ & 25 & 0.543 & 1.706 & 0.783 & 0.951 & 0.493 & 0.388 & 0.370 \\
\hline$A \sigma^{5}$ & 238 & 0.265 & 1.888 & 0.474 & 1.038 & 0.338 & 0.338 & 0.245 \\
\hline$\sigma^{6}$ & 20 & 0.615 & 1.743 & 0.747 & 0.819 & 0.449 & 0.388 & 0.387 \\
\hline$A \sigma^{6}$ & 258 & 0.306 & 1.878 & 0.500 & 1.023 & 0.348 & 0.342 & 0.259 \\
\hline$\sigma^{7}$ & 18 & 0.587 & 1.571 & 0.767 & 0.594 & 0.346 & 0.292 & 0.250 \\
\hline$A \sigma^{7}$ & 276 & 0.332 & 1.859 & 0.522 & 1.001 & 0.348 & 0.339 & 0.259 \\
\hline$\sigma^{8}$ & 15 & 0.841 & 1.566 & 0.945 & 0.579 & 0.348 & 0.268 & 0.279 \\
\hline$A \sigma^{8}$ & 291 & 0.375 & 1.845 & 0.552 & 0.983 & 0.348 & 0.336 & 0.260 \\
\hline$\sigma^{9}$ & 13 & 1.326 & 1.879 & 1.278 & 0.751 & 0.390 & 0.268 & 0.281 \\
\hline$A \sigma^{9}$ & 304 & 0.456 & 1.847 & 0.601 & 0.974 & 0.350 & 0.333 & 0.261 \\
\hline$\sigma^{10}$ & 15 & 1.206 & 1.850 & 1.186 & 0.822 & 0.389 & 0.253 & 0.230 \\
\hline$A \sigma^{10}$ & 319 & 0.515 & 1.847 & 0.641 & 0.968 & 0.352 & 0.330 & 0.259 \\
\hline$\sigma^{11}$ & 11 & 1.567 & 1.891 & 1.367 & 0.744 & 0.287 & 0.305 & 0.239 \\
\hline$A \sigma^{11}$ & 330 & 0.582 & 1.848 & 0.677 & 0.961 & 0.350 & 0.329 & 0.259 \\
\hline$\sigma^{12}$ & 9 & 1.054 & 2.067 & 1.766 & 1.022 & 0.386 & 0.213 & 0.178 \\
\hline$A \sigma^{12}$ & 339 & 0.583 & 1.854 & 0.728 & 0.963 & 0.351 & 0.326 & 0.257 \\
\hline$\sigma^{13}$ & 6 & 1.822 & 1.890 & 2.120 & 0.672 & 0.485 & 0.382 & 0.413 \\
\hline$A \sigma^{13}$ & 345 & 0.624 & 1.855 & 0.774 & 0.959 & 0.353 & 0.327 & 0.260 \\
\hline
\end{tabular}

Table III show a comparison of the results obtained for each model in the AME95-03 test. The rms deviations obtained with the GK iterative procedure are also presented. Figure 6 shows that the CLEAN algorithm improves the accuracy of all the models tested and also displays the degree of improvement achieved as a function of distance. The LDM (blue) prediction is improved by CLEAN at small distances but this improvement disappears after which the correction oscillates around the value of the model. The improvement of the LDMM (green) is more stable and has a longer range, whereas in the DZ case (red) it is apparent that beyond four units the rms of the CLEAN method ceases to improve the model predictions and oscillates around the rms deviation of the model. The nuclei region where the improvement on the prediction can be judged as reliable depends on the model and is defined as the distance where the CLEAN rms oscillates around the rms of the model, so for the models considered here the ranges are 8 units of distance for the LDM, 4 units for the DZ case, and 12 for the LDMM.

It is quite impressive to observe how the CLEAN method improves the LDM predictions, making them as accurate as the ones from the DZ model for the first units of distance. This is because the pattern obtained with the difference of the LDM is very clear and it repeats itself across the nuclear landscape, only changing its scale [see Fig. 1(a)]. On the other hand it is clear that the DZ model is significantly more difficult to improve with the CLEAN method, given its already accurate predictions.

One of the main disadvantages of the CLEAN algorithm is that its predictions depend on the model used to obtain the mass-difference pattern. Because it is known that different models can have wildly different predictions, even when they apply to masses close to the experimentally known region [22], it is important to discuss the uncertainties due to the use of different models. A consistent description of the nuclear masses would be expected in the region where the improvement to the models is considered reliable. If the predictions of the CLEAN algorithm using different models are consistent with each other then the difference between the models must be reduced after using CLEAN. Table IV shows the rms deviations between the predictions of the different models as a function of the distance defined previously both before and after using the CLEAN algorithm, which indicate that these predictions are consistent. As shown in the table, the mean deviation between the predictions of the different models is drastically reduced after using CLEAN for the three possible combinations, LDM 
TABLE IV. $\sigma^{i}$ deviations in MeV as a function of distance between the predictions of the different models considered (LDM, LDMM, and DZ) before and after applying the algorithm CLEAN. The second column shows the number of nuclei used to calculate the rms deviations.

\begin{tabular}{|c|c|c|c|c|c|c|c|}
\hline Distance & $\begin{array}{l}\text { Predicted } \\
\text { Nuclei }\end{array}$ & $\begin{array}{l}\text { LDM } \\
\text { vs. } \\
\text { LDMM }\end{array}$ & $\begin{array}{c}\text { LDM+CLEAN } \\
\text { vs. } \\
\text { LDMM+CLEAN }\end{array}$ & $\begin{array}{l}\text { LDM } \\
\text { vs. } \\
\text { DZ }\end{array}$ & $\begin{array}{c}\text { LDM+CLEAN } \\
\text { vs. } \\
\text { DZ+CLEAN }\end{array}$ & $\begin{array}{l}\text { LDMM } \\
\text { vs. } \\
\text { DZ }\end{array}$ & $\begin{array}{c}\text { LDMM+CLEAN } \\
\text { vs. } \\
\text { DZ+CLEAN }\end{array}$ \\
\hline 1 & 165 & 2.15376 & 0.197661 & 2.53302 & 0.318165 & 1.44478 & 0.294493 \\
\hline 2 & 122 & 2.14802 & 0.347624 & 2.57365 & 0.568731 & 1.60051 & 0.52335 \\
\hline 3 & 121 & 2.20684 & 0.505986 & 2.67907 & 0.877885 & 1.69461 & 0.845331 \\
\hline 4 & 120 & 2.07145 & 0.665372 & 2.71753 & 1.25192 & 1.99971 & 1.21614 \\
\hline 5 & 111 & 1.99655 & 0.791645 & 2.94985 & 1.62756 & 2.25071 & 1.58315 \\
\hline 6 & 111 & 1.97813 & 0.889578 & 3.18726 & 2.02946 & 2.52272 & 1.97478 \\
\hline 7 & 112 & 1.98505 & 0.962786 & 3.4904 & 2.42825 & 2.76453 & 2.35319 \\
\hline 8 & 114 & 1.98924 & 1.09671 & 3.84949 & 2.93075 & 3.11124 & 2.79128 \\
\hline 9 & 112 & 1.98101 & 1.18803 & 4.25154 & 3.47196 & 3.41349 & 3.25024 \\
\hline 10 & 112 & 1.95507 & 1.30185 & 4.69261 & 4.01583 & 3.82372 & 3.70363 \\
\hline
\end{tabular}

versus LDMM, LDM versus DZ, and LDMM versus DZ. This reduction is proportional to the distance to the known region, as expected. We conclude that the predictions of the CLEAN algorithm are consistent with each other, and that all of them get corrected in the same direction, at least at short range.

The results shown in this section indicate that the CLEAN extrapolations are reliable for rather short (but still sizable) ranges. It is necessary to improve the deconvolution algorithm in order to achieve more accurate predictions at larger distances. One of the basic assumptions of the CLEAN method is that the patterns to extrapolate can be modeled by a finite number of periodic harmonic components. However, the remaining patterns obtained with the difference between experimental nuclear masses and the models tested here are not periodic, but rather have a well-defined change in scale that is specified by the shell sizes (i.e., the magic numbers), so it is rather difficult to model it with harmonic components. It has been demonstrated in Ref. [23] that the CLEAN algorithm is a special case of a more general technique that is not limited by the use of a Fourier basis. Research in this direction is in progress.

\section{CONCLUSIONS}

A method to improve predictions of arbitrary nuclear mass models was presented, based on the detection and extrapolation of regularities in the pattern of differences between experimental and theoretical nuclear masses. The CLEAN algorithm was shown to be capable of detecting features in the mass-difference landscape associated with physical characteristics not included in the model. The model considers these patterns as the visible part of a concealed image. The CLEAN method is essentially a deconvolution algorithm that applies a particular methodology in order to reveal the "true" image behind a mask.

The CLEAN image reconstruction technique was applied to improve the theoretical predictions given by three different models each having a different degree of accuracy in their predictions. The models analyzed are: (a) a macroscopic LDM, (b) a macroscopic LDM with the inclusion of shellcorrection terms (LDMM), and (c) the Duflo-Zuker model. The reconstruction method has been applied to two subsets of the known nuclear landscape. In all cases important improvements in the predictions given by the different models were obtained with the CLEAN reconstruction. We analyzed the two-neutron separation energies $S_{2 n}$ and found that the CLEAN reconstruction method seems to adequately encode the physics content of the data and to reproduce in a reliable way - in both prediction tests - the presence of shell and deformation structures.

We have also tested the accuracy of the CLEAN method as a function of distance to known masses, where a special definition of distance based on the Garvey-Kelson iterative procedure has been defined. This analysis measures how the improvement of the different models by the CLEAN algorithm varies with distance to the known region. We are currently studying the use of alternative bases that may improve accuracy for regions further removed from measured masses to be able to make more reliable predictions. We believe that the CLEAN method presented here is a relatively simple method that improves predictions by nuclear mass models and that can be constantly improved by the incorporation of new measurements. It may also be susceptible to further improvements by the incorporation of procedures that can better discern the patterns present in the growing set of nuclear mass data. Finally, the same techniques can be applied to other physical observables, including nuclear radii, fission barriers, and excitation spectra, as long as enough measurements have already been made to display a pattern. Some of these questions are currently under investigation.

\section{ACKNOWLEDGMENTS}

Conversations with R. Casten and A. Zuker are gratefully acknowledged. This work was supported in part by PAPIIT-UNAM and Conacyt-Mexico. 
[1] C. E. Rolfs and W. S. Rodney, Cauldrons in the Cosmos (University of Chicago Press, Chicago, 1988).

[2] Y. Oganessian, Nature (London) 413, 122 (2001).

[3] D. Lunney, J. M. Pearson, and C. Thibault, Rev. Mod. Phys. 75, 1021 (2003).

[4] C. F. von Weizsäcker, Z. Phys. 96, 431 (1935).

[5] H. A. Bethe and R. F. Bacher, Rev. Mod. Phys. 8, 82 (1936).

[6] P. Möller, J. R. Nix, W. D. Myers, and W. J. Swiatecki, At. Data Nucl. Data Tables 59, 185 (1995).

[7] J. Duflo and A. P. Zuker, Phys. Rev. C 52, R23 (1995).

[8] S. Goriely, F. Tondeur, and J. M. Pearson, At. Data Nucl. Data Tables 77, 311 (2001).

[9] G. Audi, A. H. Wapstra, and C. Thibault, Nucl. Phys. A729, 337 (2003).

[10] J. Mendoza-Temis, A. Frank, J. G. Hirsch, J. C. López Vieyra, I. Morales, J. Barea, P. Van Isacker, and V. Velazquez, Nucl. Phys. A799, 84 (2008).

[11] G. T. Garvey and I. Kelson, Phys. Rev. Lett. 16, 197 (1966); G. T. Garvey, W. J. Gerace, R. L. Jaffe, I. Talmi, and I. Kelson, Rev. Mod. Phys. 41, S1 (1969).
[12] R. F. Casten, Nuclear Structure from a Simple Perspective (Oxford University Press, Oxford, 1990).

[13] I. O. Morales, J. C. López Vieyra, J. G. Hirsch, and A. Frank, Nucl. Phys. A828, 113 (2009).

[14] G. Audi, preprint at arXiv:nucl-ex/0302020 (2003).

[15] J. Barea, A. Frank, J. G. Hirsch, and P. Van Isacker, Phys. Rev. Lett. 94, 102501 (2005).

[16] I. Morales, A. Frank, J. C. López Vieyra, J. Barea, J. G. Hirsch, V. Velázquez, and P. Van Isacker, Int. J. Mod. Phys. E 15, 1855 (2006).

[17] J. A. Högbom, Astron. Astrophys. Suppl. 15, 417 (1974); B. G. Clark, Astron. Astrophys. 89, 377 (1980).

[18] R. Narayan and R. Nityananda, Annu. Rev. Astron. Astrophys. 24, 127 (1986).

[19] A. A. Clark, B. T. Thomas, N. W. Campbell, and P. Greenway, in British Machine Vision Conference (British Machine Vision Association, 1999), p. 193.

[20] G. Audi and A. H. Wapstra, Nucl. Phys. A595, 409 (1995).

[21] J. Mendoza-Temis et al., Nucl. Phys. A812, 28 (2008).

[22] K. Blaum, Phys. Rep. 425, 1 (2006).

[23] A. Kaup, K. Meisinger, and T. Aach, Int. J. Electron. Commun. 59, 147 (2005). 